\title{
Facial memory: Constructing familiar and unfamiliar faces
}

\author{
MARY B. YOUNT and KENNETH R. LAUGHERY \\ University of Houston, Houston, Texas 77004
}

\begin{abstract}
Sketch artists, the Identi-Kit, the Photofit, and the Field Identification System are law enforcement techniques for constructing facial images from a witness's memory. Previous research has shown that these procedures typically do not result in good facial representations of a target person. An experiment explored the effects of memory for the target on quality of the image produced with the Field Identification System. Subjects constructed four images of white male faces; two images were of highly familiar faces (e.g., husband or roommate) and two images were of unfamiliar faces viewed live for $15 \mathrm{sec}$. While significantly more time was spent generating familiar-face images, there was no difference in the quality of images for familiar vs. unfamiliar faces. The results imply that the technique has serious limitations for achieving good facial representations.
\end{abstract}

There are a number of techniques used by law enforcement agencies for constructing facial images on the basis of a witness's memory. The sketch artist and Identi-Kit are procedures widely used in the United States. Techniques commonly employed in other areas are the Photofit in Great Britain and the Minolta Montage Synthesizer in Japan. In all these procedures, a witness works with an artist or technician to construct the image. Recently, a new procedure has been developed, the Field Identification System (FIS), which enables the witness to construct an image without the involvement of another person.

Several research efforts have been reported dealing with the quality of images produced by these different techniques. Quality refers to the goodness of fit between the image and the target face. In a series of experiments, Ellis, Davies, and Shepherd (1978) and Ellis, Shepherd, and Davies (1975) explored the use of the Photofit. Laughery and Fowler (1980) studied the sketch artist and Identi-Kit techniques. Laughery, Smith, and Yount (1980) and Thompson and Laughery (Note 1) reported experiments using the FIS. Although there are technique differences in quality, one general finding seems to emerge from all of these studies; namely, the quality of images is not very good.

Why are the various techniques ineffective in producing good facial representations? There are several possible answers, including the possibility that people simply are not good at storing, retrieving, and using facial information. The techniques themselves may be limited, such as the number and variety of feature examples in the Identi-Kit, Photofit, or FIS. A third possibility is that the verbal description involved in

Requests for reprints should be sent to Kenneth R. Laughery, Psychology Department, University of Houston, Houston, Texas 77004. several of these procedures is a source of error. The latter notion seems to be supported by results reported by Davies, Shepherd, and Ellis (1978) indicating people are not good at verbally describing faces. A more recent paper by Christie and Ellis (1981), however, indicates that verbal description may be more effective than was previously thought. Of course, it is highly likely that all of these sources of error contribute to the problem of obtaining accurate images.

The experiment reported here was an attempt to shed some light on the limited-quality issue. Specifically, the purpose was to determine the extent to which the FIS is sensitive to differences in the subject's memory for the target face. The FIS was used in this study, because with the FIS, the subject constructs the image without the aid of a second person, which eliminates the verbal description.

\section{METHOD}

The study consisted of two phases, construction and rating. The construction phase dealt with the image generation part of the study. Subjects saw a target person and then constructed a facial image with the FIS. The rating phase was an experiment in which a separate group of subjects evaluated the quality of the images generated in the construction phase. Procedures for these two phases will be described separately.

\section{Construction Phase}

Subjects. Ten students at the University of Houston served as volunteer subjects.

Task. Each subject constructed six FIS composites. The first two composites were for practice to become familiar with the FIS procedure. The first image was constructed while the subject was viewing a photograph of a person. The second practice image was made from memory; that is, the subject examined a target photograph (slide) and then constructed the image. The remaining four images were made from memory. Two were of highly familiar target persons, such as a husband, longtime friend, or roommate. The other two were of unfamiliar targets seen by the subjects live for about $15 \mathrm{sec}$. 
Design. The design was simple. The one independent variable was the subject's familiarity with the target faces. Each subject constructed two familiar and two unfamiliar faces. The order of the different faces was familiar, unfamiliar, unfamiliar, familiar for half the subjects and unfamiliar, familiar, familiar, unfamiliar for the other half.

Materials and Apparatus. The FIS enables the subject to construct a facial image without the direct involvement of a second person. It consists of a book-like device with sets of horizontal page strips. The top strips contain a variety of hairstyles, the next strip section contains examples of eyes and eyebrows, the third strip set is noses and ears, and the bottom strips contain mouths and chins. The witness searches through the four strip sets and selects the appropriate hair, eyes, nose, and mouth-chin that make a composite facial image.

The photographs used for the two practice composites were front bust views of white males whose age was early to middle 20s. A watch was used to measure construction time, and the accuracy was within $5 \mathrm{sec}$.

Procedure. The subjects were run individually. They arrived at the laboratory, a small room, and were instructed as to the nature of the study. Procedures for using the FIS were described and demonstrated. Next, the subject constructed two practice images. The first image was done while a photograph of the target was in view. The second was done from memory after viewing a slide containing the target photograph for about $15 \mathrm{sec}$. Then four images were constructed, two familiar and two unfamiliar. The order was counterbalanced across subjects. The familiar faces were males who were wellknown to the subject, such as the people mentioned above. The nature of this relationship was determined in advance of accepting the person as a volunteer subject. The unfamiliar targets were college-aged white males. The target exposure consisted of having the target come to the door of the room and talk with the experimenter for approximately $15 \mathrm{sec}$. The target person moved around slightly so as to give the subject front, left side, and right side views of his face. The subject knew in advance of seeing the target that he/she would be constructing an FIS image of him.

Image construction time was measured from beginning work on the composite to its completion. Subjects were not aware the time was being recorded, and the decision to stop work on an image (regard it as completed) was up to the subject. Front bust photographs were taken of all targets, familiar and unfamiliar, for use in the rating phase.

\section{Rating Phase}

Subjects. The subjects were 15 volunteers from the University of Houston. Participation in the study resulted in extra course credit.

Task. Subjects rated the composites generated in the first phase for goodness of fit. Target photographs were projected on a screen and photocopies of the composites were stacked in front of the subject. The subject examined the photographcomposite pair and rated goodness of fit on a 6-point scale.

Materials and Apparatus. A Kodak Carousel AV-900 projector was used to display the slides. All slides were $35-\mathrm{mm}$ Ektachrome color transparencies. The photocopies were of the 60 composites produced by the subjects in the construction phase. Rating responses were recorded on answer sheets. Each sheet consisted of 33 rows corresponding to photo-composite pairs. Six columns represented the rating scale; one end of the scale was labeled "most similar" and the other end, "least similar."

Procedure. The procedure was straightforward. Subjects were seated at desks facing a screen. The composites and rating sheets were on the desks in front of them. The subjects examined each photo-composite pair and checked an appropriate column on the answer sheet in the row corresponding to that pair. The order of the pairs was randomized across subjects.
Table 1

Mean FIS Construction Times (in Minutes)

\begin{tabular}{lrr}
\hline Familiarity & First & Second \\
\hline Familiar & 12.5 & 12.5 \\
Unfamiliar & 6.8 & 8.7 \\
\hline
\end{tabular}

Table 2

Mean Ratings of Goodness of Fit

\begin{tabular}{lcc}
\hline Familiarity & First & Second \\
\hline Familiar & 3.5 & 3.8 \\
Unfamiliar & 3.7 & 3.9 \\
\hline
\end{tabular}

Note-Lower numbers indicate better fit.

\section{RESULTS}

Two dependent measures were analyzed, construction time and goodness of fit. The goodness-of-fit responses were quantified one to six, where one was most similar and six was least similar; in other words, the lower the value, the better was the image. Separate analyses of variance were carried out using these measures. The independent variables were familiarity and sequence. The latter variable was simply the first or second composite of the particular familiarity level. Hence, the analyses were 2 by 2 designs with both variables within subjects.

The mean construction times for each of the four conditions are shown in Table 1 . The effect of familiarity was significant $[F(1,9)=23.43, p<.001]$, with subjects taking longer to construct images of familiar faces. The effects of sequence and the Familiarity by Sequence interaction did not approach significance.

Mean goodness-of-fit ratings are presented in Table 2. None of the $F$ values in this analysis approached significance. Indeed, all F. values were less than 1.0.

\section{DISCUSSION}

The results of this experiment are rather startling. Typically, subjects take from $44 \%$ to $84 \%$ longer to construct an image of a highly familiar face than they do to make an unfamiliar facial image. Yet, the rated qualities of the images constructed for familiar and unfamiliar faces are virtually identical.

One implication of the results is that the real limitation in the quality of images produced with the FIS lies in the technique itself. If one accepts the very reasonable assumption that the memory representation for the familiar faces was superior to the representation for unfamiliar faces, the conclusion is warranted.

It is difficult to say precisely what the shortcomings of the FIS are. One possibility is that the feature examples are incomplete or are not good representations of real faces. Another is that the best feature matches are not easy to find. A typical comment made by subjects during construction was to express frustration at knowing what the target looked like but not being able to find a good feature match. This reaction is common in using any of the facial construction techniques. 
While the results might indicate shortcomings in the FIS "hardware," there is another explanation of the results that points to more of a procedural shortcoming. There are data in the facial memory literature that suggest that most people do not do a feature-by-feature analysis in processing facial information rather, faces are processed more holistically, or as larger units of information (Baddeley, 1979; Davies et al., 1978; Yin, 1969; Duval, Note 2). Indeed, one of the reasons the image construction techniques are so poor in general may be because they all proceed on the basis of a feature-by-feature build-up of a facial composite. If one assumes that one definition of being more familiar with a face is dealing with it as a larger unit of information, the the results of this experiment make sense. The point is that the more familiar we are with a face, the more we process it holistically (less feature processing), and hence, the less appropriate are feature composite devices such as the FIS.

The implication of this analysis is that construction techniques are needed that proceed differently. No doubt many possibilities exist. One idea that has occurred to us would be to have a set of standard prototype faces from which a witness selects a "look-alike." The procedure could then involve modifications in the look-alike prototype to better match the target. Hopefully, such approaches could make better use of more holistic information stored in the witness's memory. Whatever the explanation for the results of this experiment, one point seems clear; the outcome is not encouraging for the utility of current facial construction devices in general, and the FIS in particular.

\section{REFERENCE NOTES}

1. Thompson, B. G., \& Laughery, K. R. Facial memory: Effects of recall efforts on subsequent recognition. Paper presented at the annual meeting of the Psychonomic Society, Phoenix, Arizona, November 1979.
2. Duval, G. C. An analysis of strategies in remembering and generating faces (Report No. UHMUG-5). Houston: University of Houston, Psychology Department, 1979. (Available from K. R. Laughery, Psychology Department, University of Houston, Houston, Texas 77004.)

\section{REFERENCES}

Baddeley, A. D. Applied cognitive and cognitive applied psychology: The case of face recognition. In L. G. Nilsson (Ed.), Perspectives on memory research. Hillsdale, N.J: Erlbaum, 1979.

Christie, D. F. M., \& Ellis, H. D. Photofit constructions versus verbal descriptions of faces. Journal of Applied Psychology, 1981, 66, 358-363.

Davies, G. M., Shepherd, J. W., \& Ellis, H. D. Remembering faces: Acknowledging our limitations. Journal of the Forensic Science Society, 1978, 18, 19-24.

Ellis, H. D., Davies, G. M., \& Shepherd, J. W. A critical examination of the Photofit system for recalling faces. Ergonomicx, 1978, 21, 297-307.

Ellis, H. D., Shepherd, J. W., \& Davies, G. M. An investigation of the use of the Photofit system for recalling faces. British Journal of Psychology, 1975, 66, 29-37.

LAUGHERY, K. R., \& FowLer, R. H. Sketch artist and Identi-Kit procedures for recalling faces. Journal of Applied Psychology, 1980, 65, 307-316.

LAUGhery, K. R., Smith, V. L., \& Yount, M. B. Visual support devices: Evaluation of a new technique for constructing facial images. Proceedings of the Human Factors Society 24th Annual Meeting, Los Angeles, October 1980.

Y IN, R. K. Looking at upside-down faces. Journal of Experimental Psychology, 1969, 81, 141-145.

(Received for publication November 24, 1981.) 